\title{
The Effects of Acetazolamide on the Evaluation of Cerebral Hemodynamics and Functional Connectivity Using Blood Oxygen Level-Dependent MR Imaging in Patients with Chronic Steno-Occlusive Disease of the Anterior Circulation
}

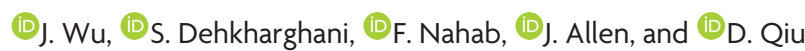

\begin{abstract}
BACKGROUND AND PURPOSE: Measuring cerebrovascular reactivity with the use of vasodilatory stimuli, such as acetazolamide, is useful for chronic cerebrovascular steno-occlusive disease. The purpose of this study was to evaluate the effects of acetazolamide on the assessment of hemodynamic impairment and functional connectivity by using noninvasive resting-state blood oxygen level-dependent MR imaging.
\end{abstract}

MATERIALS AND METHODS: A 20-minute resting-state blood oxygen level-dependent MR imaging scan was acquired with infusion of acetazolamide starting at 5 minutes after scan initiation. A recently developed temporal-shift analysis technique was applied on blood oxygen level-dependent MR imaging data before and after acetazolamide infusion to identify regions with hemodynamic impairment, and the results were compared by using contrast agent-based DSC perfusion imaging as the reference standard. Functional connectivity was compared with and without correction on the signal by using information from temporal-shift analysis, before and after acetazolamide infusion.

RESULTS: Visually, temporal-shift analysis of blood oxygen level-dependent MR imaging data identified regions with compromised hemodynamics as defined by DSC, though performance deteriorated in patients with bilateral disease. The Dice similarity coefficient between temporal-shift and DSC maps was higher before $(0.487 \pm 0.150$ by using the superior sagittal sinus signal as a reference for temporal-shift analysis) compared with after acetazolamide administration $(0.384 \pm 0.107)(P=.006$, repeated-measures ANOVA). Functional connectivity analysis with temporal-shift correction identified brain network nodes that were otherwise missed. The accuracy of functional connectivity assessment decreased after acetazolamide administration $(P=.015$ for default mode network, repeated-measures ANOVA).

CONCLUSIONS: Temporal-shift analysis of blood oxygen level-dependent MR imaging can identify brain regions with hemodynamic compromise in relation to DSC among patients with chronic cerebrovascular disease. The use of acetazolamide reduces the accuracy of temporal-shift analysis and network connectivity evaluation.

ABBREVIATIONS: $A C Z$ = acetazolamide; $B O L D=$ blood oxygen level-dependent MR imaging; DMN = default mode network; SMN = sensorimotor network; SSS = superior sagittal sinus; $T_{\max }=$ time-to-maximum of the residue function; $\mathrm{TS}=$ temporal-shift

T he measurement of cerebral perfusion can aid in the characterization of patients with cerebral ischemic diseases. ${ }^{1,2} \mathrm{Re}-$ cent studies have demonstrated that the determination of diffusion-perfusion mismatch provides a valuable paradigm for selecting a subpopulation of patients with acute stroke most likely

\section{Received May 24, 2016; accepted after revision August 20.}

From the Departments of Radiology and Imaging Sciences (J.W., S.D., J.A., D.Q.) and Neurology (F.N.), Emory University School of Medicine, Atlanta, Georgia.

Please address correspondence to Deqiang Qiu, PhD, Department of Radiology and Imaging Sciences, Emory University School of Medicine, 1364 Clifton Rd, Atlanta, GA 30322; e-mail: dqiu3@emory.edu

三 Indicates article with supplemental on-line table.

Indicates article with supplemental on-line photos.

http://dx.doi.org/10.3174/ajnr.A4973 to benefit from reperfusion therapies. ${ }^{3-8}$ However, MR perfusion imaging is typically based on DSC with bolus injection of a gadolinium-based contrast agent. ${ }^{5,8-10}$ Although the risk of nephrogenic systemic fibrosis associated with the use of gadoliniumbased contrast agents may be minimized through renal function screening, there are recent concerns about chronic deposition of gadolinium in the brain. ${ }^{11}$ The use of a contrast agent may furthermore preclude repeat perfusion scans in the same session, ${ }^{12}$ which are needed in clinical settings such as the evaluation of cerebrovascular reactivity.

The development of noninvasive approaches without the need for contrast agent administration can provide useful alternatives. Although arterial spin-labeling is a noninvasive method for measuring $\mathrm{CBF}$, it is prone to errors in regions with a long arterial 
transit time of blood, ${ }^{12-14}$ which is particularly problematic in patients with steno-occlusive disease. Recently, temporal-shift (TS) analysis of the resting-state blood oxygen level-dependent MR imaging (BOLD) signal, which is sensitive to local blood flow and oxygen metabolism, ${ }^{15}$ has been shown to depict regions with cerebrovascular impairment in acute stroke and chronic cerebral hypoperfusion. ${ }^{16-18}$ In addition, compared with the measurement of hemodynamic parameters, the assessment of the functional status of such hypoperfused brain is underinvestigated. A growing body of work supports resting-state BOLD signal possibly being used to evaluate functional brain networks. ${ }^{19-23}$ Leveraging different aspects of the same BOLD acquisition, simultaneous assessment of cerebral hemodynamics and functional connectivity therefore becomes an attractive application of resting-state BOLD.

Traditionally, cerebrovascular reactivity has been an important measure in patients with chronic steno-occlusive disease. The measurement of cerebrovascular reactivity is performed by quantifying cerebrovascular responses to vasodilatory stimuli, such as the administration of acetazolamide (ACZ) or inhalation of air with increased $\mathrm{CO}_{2}$ concentration (eg, 5\%). ${ }^{24-26}$ Examining the effects of vasodilatory stimuli on TS and functional connectivity analyses may shed light on their physiologic basis and allow development of an operationalized approach to their evaluation. In this study, we aimed to assess the effects of ACZ on the evaluations of hemodynamic impairment and functional brain connectivity by using resting-state BOLD in patients with chronic steno-occlusive disease of the anterior circulation. We hypothesized that TS analysis of BOLD data could identify regions with hemodynamic compromise in patients with chronic cerebrovascular disease, similar to those shown in acute stroke and Moyamoya disease. We further hypothesized that the use of ACZ would affect the results of TS and functional connectivity analyses due to its alteration in neurovascular coupling.

\section{MATERIALS AND METHODS \\ Participants}

Fourteen patients with chronic steno-occlusive disease of the anterior circulation (mean age, 48 years; range, 31-70 years; 3 men, 11 women) were included in this study. These included 4 patients with idiopathic Moyamoya disease (3 with bilateral disease), 7 patients with atherosclerotic occlusion of the MCA or ICA (2 with bilateral disease), and 3 patients with unilateral atherosclerotic high-grade stenosis of the ICA (see the On-line Table for details). The study was approved by our institutional review board; and all patients underwent specialized acetazolamide-challenge MR imaging. Ten had follow-up scans the next day without ACZ administration.

\section{Data Acquisition and Preprocessing}

MR imaging was performed at 3T (Tim Trio; Siemens, Erlangen, Germany). BOLD images were acquired by using a gradient-echo EPI sequence: $\mathrm{TR} / \mathrm{TE}=2000 / 30 \mathrm{~ms}$, flip angle $=78^{\circ}, \mathrm{FOV}=$ $220 \times 220 \mathrm{~mm}^{2}$, matrix $=64 \times 64$, section thickness $=4 \mathrm{~mm}, 30$ sections. The entire BOLD scan spanned 20 minutes. At 5 minutes after the initiation of the scan, $1 \mathrm{~g}$ of ACZ dissolved in $10 \mathrm{~mL}$ of normal saline was slowly infused intravenously for 3-5 minutes without interruption of the scanning session.

At the conclusion of the BOLD acquisition, DSC imaging was performed by using a gradient-echo EPI sequence $(\mathrm{TR}=1500 \mathrm{~ms}$, $\mathrm{TE}=40 \mathrm{~ms}$, flip angle $=60^{\circ}, \mathrm{FOV}=240 \times 240 \mathrm{~mm}^{2}$, matrix $=$ $128 \times 128$, section thickness $=5 \mathrm{~mm}, 19$ sections) with the injection of $0.1 \mathrm{mmol} / \mathrm{kg}$ of gadobenate dimeglumine (MultiHance; Bracco Diagnostics, Princeton, New Jersey) delivered by a power injector at $4 \mathrm{~mL} / \mathrm{s}$ through an antecubital intravenous access and followed by a normal saline flush at the same rate. Automated arterial input function and venous output function detection, followed by a delay-insensitive deconvolution with a regularization threshold of $15 \%$ of the maximum singular value, ${ }^{27}$ were implemented to generate perfusion maps, including CBF, CBV, MTT, and the time-to-maximum of the residue function $\left(\mathrm{T}_{\max }\right)$. The perfusion maps were further spatially normalized to standard Montreal Neurological Institute space.

Additionally, T1-weighted MPRAGE imaging (TR $=1900 \mathrm{~ms}$, $\mathrm{TE}=3.52 \mathrm{~ms}$, flip angle $=9^{\circ}, \mathrm{FOV}=216 \times 256 \mathrm{~mm}^{2}$, matrix $=$ $216 \times 256$, section thickness $=1 \mathrm{~mm}, 176$ ) was performed for anatomic localization.

Preprocessing of BOLD images was performed by using SPM8 software (http://www.fil.ion.ucl.ac.uk/spm/software/spm8). The first and last 5 minutes of data (150 volumes) were used for analysis, representing data acquired before (pre-ACZ) and after (post-ACZ) administration of ACZ, respectively. The last 5 minutes of images were used for post-ACZ assessment because we found that the ACZ effect plateaued approximately 10 minutes after the initiation of infusion. For both pre-ACZ and post-ACZ data, after removal of the first 10 volumes, BOLD images were corrected for the timing of the section acquisition, realigned to the mean image, normalized to the Montreal Neurological Institute space, resampled to $3-\mathrm{mm}$ isotropic voxel size, and spatially smoothed with a Gaussian kernel of 6-mm full width at half maximum. After removal of the linear temporal trend from the processed images, we regressed out the effects of head motion by using estimates (6 parameters) from the above realignment step as confounding factors. Last, the data were bandpass-filtered to retain signal components with temporal frequency between 0.01 and $0.1 \mathrm{~Hz}$.

\section{Temporal-Shift Maps}

TS maps were calculated from the BOLD data by determining, for each voxel, the temporal offset that maximizes the correlation coefficient between the time-shifted ( -6 TR to +6 TR; ie, -12 seconds to +12 seconds) reference signal and the temporal signal of each voxel (On-line Fig 1). The temporal offset was then assigned as the value of the respective voxel in the TS map. We considered 2 options of reference signals: the global mean signal and the average time-series over an ROI within the superior sagittal sinus (SSS). The global mean signal was obtained by calculating the mean temporal signal across the entire brain, including contributions from GM, WM, and CSF. To obtain the SSS reference signal, we calculated a temporal SD map of the BOLD signal to reflect the magnitude of spontaneous signal fluctuation in each voxel. Because the SSS has $100 \%$ blood volume compared with a maximum of $5 \%$ blood volume in brain tissue, the BOLD signal of the SSS has a large variance compared with other regions. An ROI was therefore 


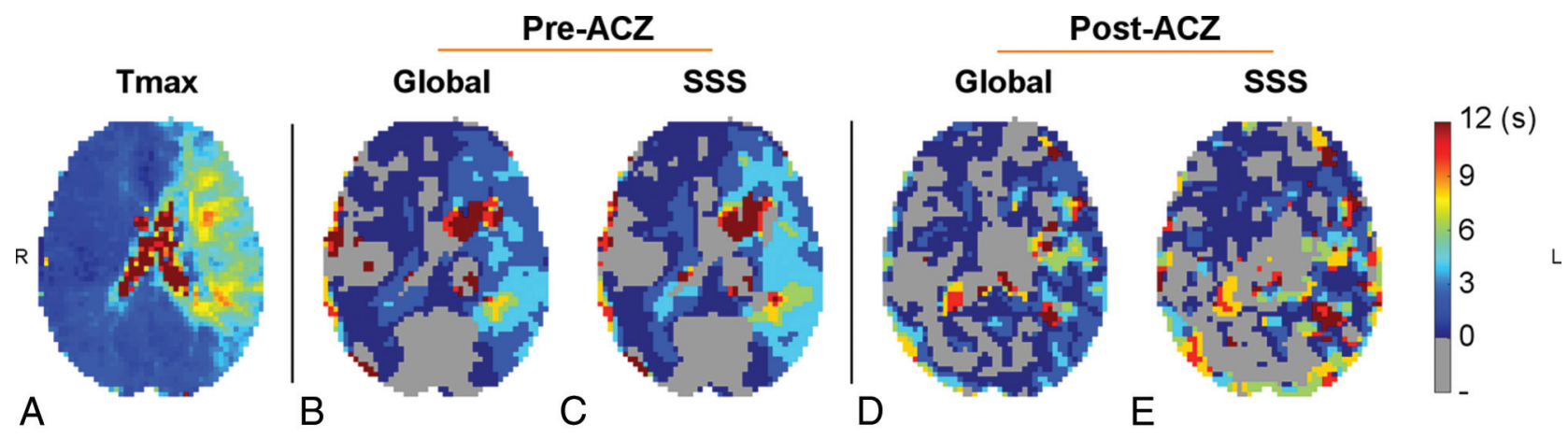

FIG 1. DSC $T_{\max }(A)$ and temporal-shift maps $(B-E)$ derived from resting-state BOLD data by using the global mean signal $(B$ and $D)$ and the superior sagittal sinus $(C$ and $E$ ) as references, as well as before $(B$ and $C$ ) and after $(D$ and $E$ ) acetazolamide administration in a patient with occlusion of the left ICA. Visually, TS maps exhibited a global resemblance to $T_{\max }$ maps. This agreement decreased after ACZ administration. Compared with using the global signal as reference, TS maps generated by using the SSS signal as the reference signal showed a better agreement with $\mathrm{T}_{\max }$ maps. The images are in radiologic convention.

manually placed in a brain area with a high SD in the SSS without including adjacent brain tissues (4 voxels for each subject).

While the success of TS analysis depends on the optimal selection of a reference signal (ie, the identification of "normal" tissues), this information could, in turn, be provided by the results of TS analysis. We therefore proposed an iterative approach to optimize the computation of TS maps by improving estimation of the reference signal as shown below. The average time-series of all voxels with a zero time delay (offset $=0$ second) from the previous iteration was calculated and used as the new reference signal for the next iteration. The mean TS value over the whole brain was also calculated. The iteration was repeated until convergence in that the absolute difference of mean values between the TS maps in the successive iterations was lower than 0.001 second.

\section{Functional Connectivity Analysis}

The default mode network (DMN) and sensorimotor network (SMN) were identified and evaluated by using a seed-based functional connectivity analysis method on the BOLD data. ${ }^{19,21,28}$ Using a functional ROI atlas, ${ }^{29}$ we defined the seed region for the $\mathrm{DMN}$ in the precuneus/posterior cingulate cortex: Montreal Neurological Institute coordinate $=(0,-56,28)$, radius $=10 \mathrm{~mm}$. The seed ROI for the SMN was placed in the precentral gyrus of the unaffected or less affected hemisphere: Montreal Neurological Institute coordinate $=( \pm 44,-16,46)$, radius $=6 \mathrm{~mm}$. For each network, the average temporal signal in the seed ROI of the preprocessed images was calculated, and then the Pearson correlation coefficient $r$ with this seed temporal signal was calculated for all brain voxels to generate the functional connectivity maps. To evaluate the effect of correction by using temporal-shift information from the TS analysis on functional connectivity analysis, we shifted the temporal signal of each voxel according to the value in the TS map; the functional connectivity analysis was then repeated. This correction is termed "TS correction."

\section{Image and Statistical Analysis}

A mixed-effects model was used to study the correlation between the TS and $\mathrm{T}_{\text {max }}$ maps and the effect of ACZ administration (pre$A C Z$ versus post-ACZ) on this correlation. The mean values of BOLD TS and $\mathrm{T}_{\max }$ were calculated in regions with $\mathrm{T}_{\max }$ values binned in different ranges ( $0-1$ second, $1-2$ seconds, $2-3$ seconds,
3-4 seconds, $4-5$ seconds, $5-6$ seconds, and $>6$ seconds). The linear relationship between the mean TS and $\mathrm{T}_{\max }$ was estimated by modeling subject-specific slope as a random effect.

To further assess the potential clinical value of the TS map, we evaluated the spatial overlap between regions with perfusion deficits as defined by the TS and DSC $\mathrm{T}_{\max }$ maps by using the Dice similarity coefficient. ${ }^{18}$ The Dice coefficient between regions A and $B$ was defined as the following: Similarity $=2|A \cap B| /(|A|+$ $|B|)$, where $|A \cap B|$ represents the area of the overlapped region between $A$ and $B$ and $|A|$ and $|B|$ represent the areas of $A$ and $B$, respectively. Because the threshold of $\mathrm{T}_{\max }$ for quantifying pathologic tissue volumes is yet to be established in patients with chronic cerebrovascular disease, we used 4 seconds as the threshold, which was an established threshold for estimating hypoperfused tissue volumes in patients with acute stroke. ${ }^{27,30}$ Additional analysis by using $\mathrm{T}_{\max }>3$ seconds as a threshold was also performed to improve the sensitivity for identifying areas with hemodynamic compromise. The optimal threshold for a TS map that maximized its Dice similarity with its respective $T_{\max }$ map was derived by varying the threshold from -12 to 12 seconds with increments of 2 seconds (ie, TR). The values of the Dice similarity were compared by using 2-way repeated-measures ANOVA to test the effect of ACZ administration (pre-ACZ versus post-ACZ) and the choice of reference signal (the global signal versus the SSS signal) on TS analysis.

The Dice similarity coefficient between patient functional networks and templates of brain networks derived from healthy-subject resting-state $\mathrm{BOLD}$ data ${ }^{29}$ was calculated to assess the effects of TS correlation and ACZ on functional connectivity assessment. Twoway repeated-measures ANOVA was performed to test whether ACZ administration (pre-ACZ versus post-ACZ) and TS correction (original versus TS-corrected analysis) influenced the assessment of functional connectivity.

\section{RESULTS}

The mixed-effects model showed significant correlation between the TS and $\mathrm{T}_{\max }$ maps when using either the global signal or the SSS as the reference signal for calculating TS maps $(P<.001$ for both tests). The slopes of change in TS with respect to $\mathrm{T}_{\max }$ were significantly lower after ACZ administration when using the 
global signal $(P=.001)$ and SSS $(P=.026)$ as the reference signal, respectively (On-line Fig 2 ).

Figure 1 and On-line Fig 3 show the comparison of restingstate BOLD TS maps and DSC $\mathrm{T}_{\max }$ maps in representative patients. Brain regions with a long $\mathrm{T}_{\max }$ were associated with a positive TS value in the temporal-shift analysis (a delay in the time course with respect to the reference signal). The On-line Table shows the Dice similarity coefficient between the $\mathrm{T}_{\max }$ and TS maps in defining brain regions with compromised perfusion for each patient. Using $\mathrm{T}_{\max }>4$ seconds as a threshold for compro-
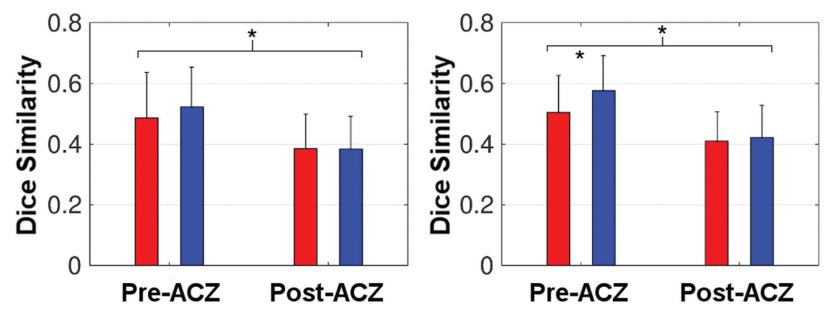

A

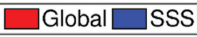

B

$\square$ Global $\square$ SSS

FIG 2. The mean Dice similarity coefficient between temporal-shift and $T_{\max }$ maps by using $T_{\max }>4$ seconds $(A)$ and $T_{\max }>3$ seconds $(B)$ as criteria for defining hemodynamic compromise, respectively. TS maps were derived from resting-state BOLD by using either the global signal (red bars) or the superior sagittal sinus signal (blue bars) as the reference. When we used $\mathrm{T}_{\max }>4$ seconds as the threshold, a lower Dice similarity between $T_{\max }$ and TS maps was found after acetazolamide administration (post-ACZ) compared with that before acetazolamide (pre-ACZ). When using $\mathrm{T}_{\max }>3$ seconds as the threshold, TS maps obtained with the SSS signal as the reference showed greater similarity to $T_{\max }$ maps than those using the global signal in the pre$A C Z$ condition. Compared with the pre-ACZ results, the post- $A C Z$ results demonstrated lower similarity. Error bars represent the SD of the mean. Asterisks indicate significant differences as determined by 2-way repeated-measures ANOVA with a post hoc Bonferroni-corrected paired $t$ test.

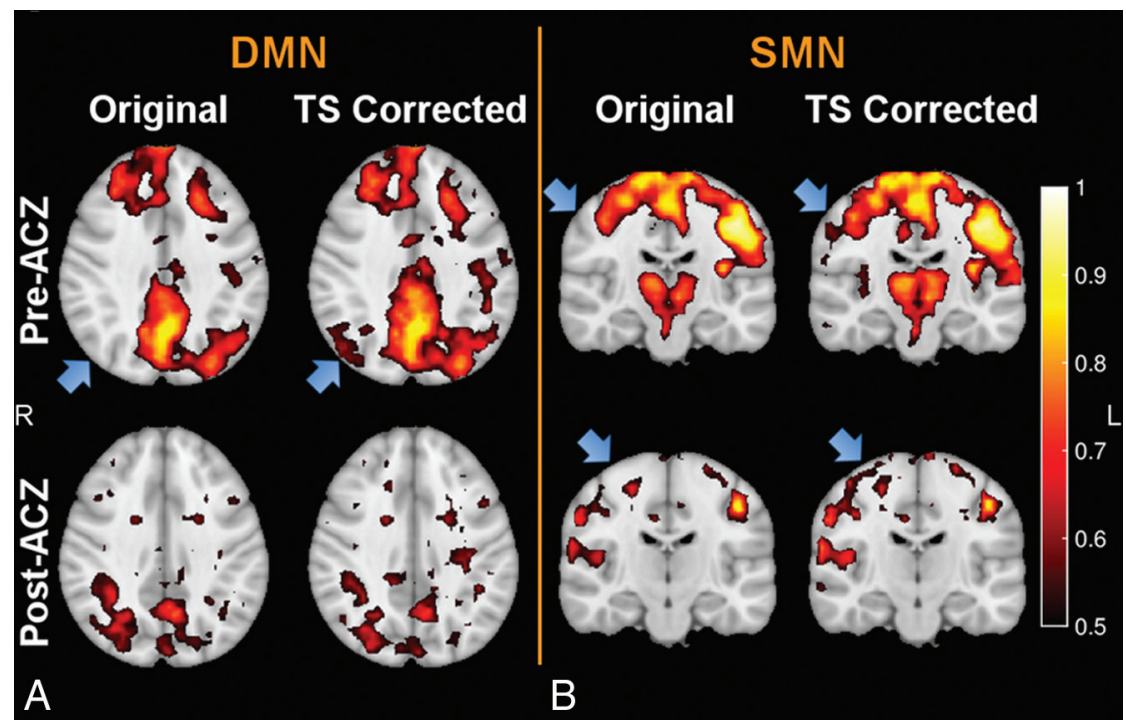

FIG 3. The default mode network $(A)$ and the sensorimotor network ( $B$ ) overlaid on T1-weighted images before (upper row) and after (lower row) acetazolamide administration, as well as without and with temporal-shift correction in a patient with occlusion of the right MCA. Although functional connectivity changed after ACZ challenge, the DMN and SMN could be still identified. New network nodes were uncovered in the hypoperfused areas after the correction of time delay derived from TS analysis (see the blue arrows in the original and TS-corrected networks). In general, these regions could be observed in the corresponding networks of healthy subjects. The images are in radiologic convention. mised perfusion, 2-way repeated-measures ANOVA showed that after ACZ administration, the Dice similarity coefficient between TS and $\mathrm{T}_{\max }$ maps was significantly reduced compared with that before ACZ administration ( $P=.006$ ) (Fig $2 A$; see the On-line Table for detailed statistics).

When we used $\mathrm{T}_{\max }>3$ seconds as a threshold for compromised perfusion, significant effects were found related to the choice of different reference signals $(P=.008)$, ACZ administration $(P=.003)$, and the interaction between them $(P=.020)$ on the similarity coefficient between TS and $\mathrm{T}_{\max }$ maps (Fig $2 B$ and On-line Table). Post hoc Bonferroni-corrected paired $t$ tests showed the following: 1) before ACZ administration, TS maps derived by using SSS as the reference signal demonstrated higher similarity with $\mathrm{T}_{\max }$ maps, compared with those using the global signal as the reference signal $(P<.001)$; and 2$)$ lower similarity between $\mathrm{T}_{\max }$ and TS maps was found after ACZ administration compared with before $\mathrm{ACZ}$ administration when using either the global signal $(P=.011)$ or SSS $(P=.002)$ as the reference signal (Fig $2 B$ and On-line Table). For the above analysis, the DSC perfusion after ACZ administration was used. The same analysis was repeated in a subset of 10 patients who underwent a second-day MR imaging examination without the administration of ACZ, and in these cases, the pre-ACZ second-day $\mathrm{T}_{\max }$ was used. Similar results were obtained (On-line Fig 4).

The default mode network and sensorimotor network of representative patients are shown in Fig 3 and On-line Fig 5. Although the results of functional connectivity changed after ACZ administration, both the DMN and SMN remained identifiable. TS correction uncovered new network nodes in areas with hemodynamic compromise in some patients. In general, these emerged nodes could be observed in the corresponding functional networks of healthy subjects. Temporal-shift information obtained from TS analysis by using the SSS signal as the reference was used to correct the BOLD signal for functional connectivity analysis because of its better performance in delineating regions with hemodynamic impairment.

Two-way repeated-measures ANOVA showed significant effects of TS correction on the functional connectivity assessment of the DMN $(P<.001)$ and the SMN $(P=.024)$ (Fig 4). Accuracy in the assessment of both functional networks (as measured by the overlap with template network masks) increased after correction of the signal by using time-shift estimates from the TS analysis. Assessment accuracy decreased after ACZ administration, with statistically significant effects for the DMN $(P=.015)$ and a trend toward significance for the $\operatorname{SMN}(P=.080)$.

\section{DISCUSSION}

The present study demonstrated that temporal-shift maps derived from noninvasive resting-state BOLD scans could identify brain regions with abnormal per- 


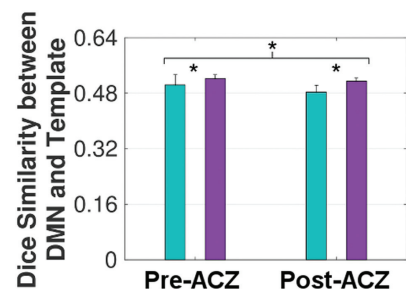

A
B

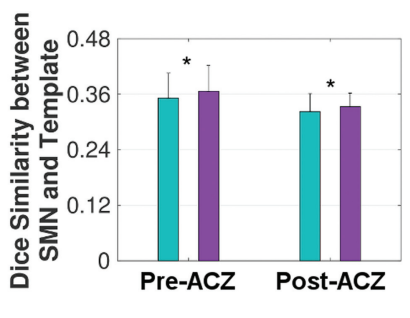

Original $\square$ TS Corrected
FIG 4. The accuracy of functional connectivity assessment, measured by the Dice similarity coefficient between functional networks and network templates, for default mode $(A)$ and sensorimotor $(B)$ networks. The assessment accuracy of both functional networks increased after temporal-shift correction. The assessment accuracy decreased after acetazolamide administration for the DMN, while a trend toward significance was found for the SMN. Error bars represent the SD of the mean. Asterisks indicate significant differences as determined by 2-way repeated-measures ANOVA.

fusion in patients with chronic steno-occlusive disease of the anterior circulation. The computed temporal-shift maps qualitatively and quantitatively resembled $\mathrm{T}_{\max }$ maps derived from contrast agent-based DSC perfusion imaging. Acetazolamide administration reduced the similarity between temporal-shift maps and $\mathrm{T}_{\max }$ maps. The performance of temporal-shift analysis in identifying regions with hemodynamic impairment deteriorated in patients with bilateral disease. Functional connectivity analysis on the default mode and sensorimotor networks showed impaired diminished connectivity in some patients, possibly due to impaired neural activity or compromised neurovascular coupling. The correction of temporal signals by using information derived from temporal-shift analysis showed mixed results, with recovery of otherwise missing network nodes in some but not all patients. The use of ACZ reduced the overall accuracy in the identification of resting-state networks.

The evaluation of cerebral perfusion plays an important role in identifying patients with acute stroke who are most likely to benefit from perfusion restoration to ischemic tissues ${ }^{3-5}$ and has traditionally relied on MR imaging or CT perfusion imaging with the use of a contrast agent. Although the quantitative value of DSC in evaluating patients with chronic cerebrovascular disease remains to be established, it is qualitatively useful in forming an overall impression of the pathologic status. The requirement of exogenous contrast agent application could be a limitation due to the potential for adverse effects in patients with severe renal impairment as well as recent concerns about gadolinium deposition in the brain and other tissues; this therefore potentially precludes the use of DSC perfusion imaging in applications in which repeated scans are needed. The development of noninvasive methods free of contrast agent provides an alternative in situations in which the use of contrast agent is contraindicated. Our results showed that maps from temporal-shift analysis resembled DSC perfusion $\mathrm{T}_{\max }$ maps both visually and quantitatively. This finding provides corroborative evidence that spontaneous fluctuations in the BOLD signal contain information that can be isolated to approximate cerebral perfusion impairment, in addition to neuronal activity, as demonstrated in other patient populations. ${ }^{16-18}$ However, differences between TS and $\mathrm{T}_{\max }$ maps were also observed. The exact mechanism for potential discrepancies is not completely understood, but the differences are not surprising because TS analysis and DSC perfusion imaging likely reflect different aspects of the same pathophysiologic process. Further development of the technique and validation within larger cohorts are necessary to further establish the nature of this relation and to further assess TS maps as an adjunct or potential alternative to DSC perfusion imaging in patients with neurovascular disease.

Two options of the reference signals were considered in the temporal-shift analysis: the global mean signal and the signal from the SSS. While both approaches produced TS maps comparable with $\mathrm{T}_{\max }$ maps, a higher similarity was observed between $\mathrm{T}_{\max }$ maps and TS maps obtained by using the SSS signal as the reference. This suggests that the largely fluctuating signal in the venous sinus and the iterative strategy ensured a better assessment of hemodynamic compromise. Furthermore, while global signal can be easily calculated even without operator input, the accuracy of the reference may be contaminated by inclusion of neural activity $^{31}$ and the hypoperfused brain regions. The problem became particularly apparent when hypoperfused regions were large, such as in patients with bilateral steno-occlusive disease. We also found that the time delay evaluated by using the global signal as a reference tended to be smaller compared with that calculated with the SSS as reference. This finding could be because the global signal showed a time lag from normal areas because of the contribution from the hypoperfused areas, which is weighted by the volumes of these areas. In contrast, although the SSS receives blood from hypoperfused areas as well, the contribution from these areas is weighted by their blood flow; and because of the reduced blood flow in these areas, the contribution from them is smaller.

When we used the temporal delay information calculated from resting-state BOLD data to correct for the functional connectivity analysis of the DMN and the SMN, new areas of both functional networks were found, particularly in regions with hemodynamic compromise. Such areas generally emerged in locations where brain networks of healthy participants typically reside. These findings suggest caution when evaluating functional connectivity in patients with cerebrovascular compromise. For example, an apparent reduction in connectivity could be recovered by TS correction and might not be caused by changes in the underlying neuronal activity but rather by changes in neurovascular coupling.

It has been reported that neuronal function and underlying neurovascular coupling could not be correctly evaluated by BOLD MR imaging in the setting of neurovascular ischemia, purportedly due to the uncoupling of the positive relationship between CBF and BOLD responses under physiologic baseline conditions. ${ }^{26,32,33}$ In this study, changes in the detected DMN and SMN were observed after ACZ administration, though spontaneous neural activity should be unaltered without explicit external neural stimuli. Our finding consequently provides further evidence for the altered assessment of neuronal function of BOLD MR imaging after a vasodilatory challenge. Also, TS analysis needs to be applied to evaluate neuronal activity by using BOLD MR imaging in patients with steno-occlusive disease, due to the possibility of compromised neurovascular coupling. Moreover, we found that TS maps exhibited a decreased similarity with $\mathrm{T}_{\max }$ after ACZ administration. This is consistent with previous findings showing that BOLD responses are reduced in task-based 
fMRI after ACZ challenge. ${ }^{26,33}$ Although ACZ is useful for interrogating cerebrovascular reactivity, there should be caution in interpreting the results of functional connectivity and TS analyses in BOLD studies after ACZ administration.

Several issues remain to be addressed in future studies. First, while the SSS reference approach provided a good estimate of hemodynamic compromise, the selection of ROIs within the SSS could affect the results. An algorithm for automatic determination of the SSS ROI is required to derive optimal results. Second, a relatively low spatial (voxel size $=3.4 \times 3.4 \times 4 \mathrm{~mm}^{3}$ ) and temporal resolution ( $\mathrm{TR}=2$ seconds) BOLD sequence was used in the current study. Higher spatial and temporal resolutions can be achieved via multiband acquisitions, thereby improving the accuracy of the assessment of hemodynamic compromise, similar to that found in resting-state fMRI. ${ }^{34,35}$ Third, although there is variability in the TS correction in unveiling otherwise occult nodes in functional networks, whether this reflects the severity of functional compromise is not clear. Further studies are required to systematically investigate whether functional connectivity with temporal-shift correction is associated with functional/cognitive symptoms and whether this information can be used to better predict functional recovery among these patients.

\section{CONCLUSIONS}

Temporal-shift analysis of non-contrast agent-based restingstate BOLD data can delineate brain areas with hemodynamic compromise as measured by DSC $\mathrm{T}_{\max }$ maps in patients with chronic cerebrovascular disease, though the performance of temporal-shift analysis deteriorates in patients with bilateral disease. Temporal-shift correction recovers nodes of functional brain networks in some hypoperfused areas. The use of ACZ changes the effectiveness of temporal-shift analysis in evaluating cerebral hemodynamic compromise as well as functional connectivity analysis from resting-state BOLD data.

Disclosures: Fadi Nahab_UNRELATED: Employment: The Joint Commission; Expert Testimony: provided legal counsel as an expert witness on cases involving stroke patients; Payment for Lectures including Service on Speakers Bureaus: Medtronic, Genzyme; Payment for Development of Educational Presentations: Imedex. Deqiang Qiu-UNRELATED: Grants/Grants Pending: Siemens.* *Money paid to the institution.

\section{REFERENCES}

1. Merino JG, Warach S. Imaging of acute stroke. Nat Rev Neurol 2010; 6:560-71 CrossRef Medline

2. Dani KA, Thomas RGR, Chappell FM, et al; Translational Medicine Research Collaboration Multicentre Acute Stroke Imaging Study. Computed tomography and magnetic resonance perfusion imaging in ischemic stroke: definitions and thresholds. Ann Neurol 2011; 70:384-401 CrossRef Medline

3. Latchaw RE, Yonas H, Hunter GJ, et al; Council on Cardiovascular Radiology of the American Heart Association. Guidelines and recommendations for perfusion imaging in cerebral ischemia: a scientific statement for healthcare professionals by the writing group on perfusion imaging, from the Council on Cardiovascular Radiology of the American Heart Association. Stroke 2003;34:1084-104 CrossRef Medline

4. Marks MP, Lansberg MG, Mlynash M, et al; DEFUSE Investigators. Angiographic outcome of endovascular stroke therapy correlated with MR findings, infarct growth, and clinical outcome in the DEFUSE 2 trial. Int J Stroke 2014;9:860-65 CrossRef Medline
5. Lansberg MG, Straka M, Kemp S, et al; DEFUSE 2 study investigators. MRI profile and response to endovascular reperfusion after stroke (DEFUSE 2): a prospective cohort study. Lancet Neurol 2012;11: 860-67 CrossRef Medline

6. Saver JL, Goyal M, Bonafe A, et al; SWIFT PRIME Investigators. Stent-retriever thrombectomy after intravenous t-PA vs. t-PA alone in stroke. $N$ Engl J Med 2015;372:2285-95 CrossRef Medline

7. Jovin TG, Chamorro A, Cobo E, et al; REVASCAT Trial Investigators. Thrombectomy within $\mathbf{8}$ hours after symptom onset in ischemic stroke. N Engl J Med 2015;372:2296-306 CrossRef Medline

8. Campbell BCV, Christensen S, Levi CR, et al. Comparison of computed tomography perfusion and magnetic resonance imaging perfusion-diffusion mismatch in ischemic stroke. Stroke 2012;43: 2648-53 CrossRef Medline

9. Saver JL, Goyal M, Bonafe A, et al; SWIFT PRIME Investigators. Solitaire $^{\mathrm{TM}}$ with the Intention for Thrombectomy as Primary Endovascular Treatment for Acute Ischemic Stroke (SWIFT PRIME) trial: protocol for a randomized, controlled, multicenter study comparing the Solitaire revascularization device with IV tPA with IV tPA alone in acute ischemic stroke. Int J Stroke 2015;10:439-48 CrossRef Medline

10. Østergaard L. Principles of cerebral perfusion imaging by bolus tracking. J Magn Reson Imaging 2005;22:710-17 CrossRef Medline

11. McDonald RJ, McDonald JS, Kallmes DF, et al. Intracranial gadolinium deposition after contrast-enhanced MR imaging. Radiology 2015;275:772-82 CrossRef Medline

12. Ovadia-Caro S, Margulies DS, Villringer A. The value of resting-state functional magnetic resonance imaging in stroke. Stroke 2014;45: 2818-24 CrossRef Medline

13. Petersen ET, Zimine I, Ho YC, et al. Non-invasive measurement of perfusion: a critical review of arterial spin labelling techniques. $\mathrm{BrJ}$ Radiol 2006;79:688-701 CrossRef Medline

14. Bokkers RP, Hernandez DA, Merino JG, et al; National Institutes of Health Stroke Natural History Investigators. Whole-brain arterial spin labeling perfusion MRI in patients with acute stroke. Stroke 2012;43:1290-94 CrossRef Medline

15. Villringer A, Dirnagl U. Coupling of brain activity and cerebral blood flow: basis of functional neuroimaging. Cerebrovasc Brain Metab Rev 1995;7:240-76 Medline

16. Christen T, Jahanian $\mathrm{H}, \mathrm{Ni}$ WW, et al. Noncontrast mapping of arterial delay and functional connectivity using resting-state functional MRI: a study in Moyamoya patients. J Magn Reson Imaging 2015;41:424-30 CrossRef Medline

17. Amemiya S, Kunimatsu A, Saito N, et al. Cerebral hemodynamic impairment: assessment with resting-state functional MR imaging. Radiology 2014;270:548-55 CrossRef Medline

18. Lv Y, Margulies DS, Cameron Craddock R, et al. Identifying the perfusion deficit in acute stroke with resting-state functional magnetic resonance imaging. Ann Neurol 2013;73:136-40 CrossRef Medline

19. Fox MD, Raichle ME. Spontaneous fluctuations in brain activity observed with functional magnetic resonance imaging. Nat Rev Neurosci 2007;8:700-11 CrossRef Medline

20. Smith SM, Fox PT, Miller KL, et al. Correspondence of the brain's functional architecture during activation and rest. Proc Natl Acad Sci U S A 2009;106:13040-45 CrossRef Medline

21. van den Heuvel MP, Hulshoff Pol HE. Exploring the brain network: a review on resting-state fMRI functional connectivity. Eur Neuropsychopharmacol 2010;20:519-34 CrossRef Medline

22. Chang TY, Huang KL, Ho MY, et al. Graph theoretical analysis of functional networks and its relationship to cognitive decline in patients with carotid stenosis. J Cereb Blood Flow Metab 2016;36: 808-18 Medline

23. Cheng HL, Lin CJ, Soong BW, et al. Impairments in cognitive function and brain connectivity in severe asymptomatic carotid stenosis. Stroke 2012;43:2567-73 CrossRef Medline

24. Gupta A, Chazen JL, Hartman M, et al. Cerebrovascular reserve and stroke risk in patients with carotid stenosis or occlusion: a systematic review and meta-analysis. Stroke 2012;43:2884-91 CrossRef Medline 
25. Vagal AS, Leach JL, Fernandez-Ulloa M, et al. The acetazolamide challenge: techniques and applications in the evaluation of chronic cerebral ischemia. AJNR Am J Neuroradiol 2009;30:876-84 CrossRef Medline

26. Siero JC, Hartkamp NS, Donahue MJ, et al. Neuronal activation induced BOLD and CBF responses upon acetazolamide administration in patients with steno-occlusive artery disease. Neuroimage 2015;105:276-85 CrossRef Medline

27. Straka M, Albers GW, Bammer R. Real-time diffusion-perfusion mismatch analysis in acute stroke. J Magn Reson Imaging 2010;32: 1024-37 CrossRef Medline

28. Biswal B, Yetkin FZ, Haughton VM, et al. Functional connectivity in the motor cortex of resting human brain using echo-planar MRI. Magn Reson Med 1995;34:537-41 CrossRef Medline

29. Shirer WR, Ryali S, Rykhlevskaia E, et al. Decoding subject-driven cognitive states with whole-brain connectivity patterns. Cereb Cortex 2012;22:158-65 CrossRef Medline

30. Albers GW, Thijs VN, Wechsler L, et al; DEFUSE Investigators. Magnetic resonance imaging profiles predict clinical response to early reperfusion: the diffusion and perfusion imaging evaluation for understanding stroke evolution (DEFUSE) study. Ann Neurol 2006; 60:508-17 CrossRef Medline

31. Amemiya S, Takao H, Hanaoka S, et al. Global and structured waves of rs-fMRI signal identified as putative propagation of spontaneous neural activity. Neuroimage 2016;133:331-40 CrossRef Medline

32. Blicher JU, Stagg CJ, O'Shea J, et al. Visualization of altered neurovascular coupling in chronic stroke patients using multimodal functional MRI. J Cereb Blood Flow Metab 2012;32:2044-54 CrossRef Medline

33. Brown GG, Eyler Zorrilla LT, Georgy B, et al. BOLD and perfusion response to finger-thumb apposition after acetazolamide administration: differential relationship to global perfusion. J Cereb Blood Flow Metab 2003;23:829-37 Medline

34. Preibisch C, Castrillón G JG, Bührer M, et al. Evaluation of multiband EPI acquisitions for resting state fMRI. PLoS One 2015;10: e0136961 CrossRef Medline

35. Feinberg DA, Moeller S, Smith SM, et al. Multiplexed echo planar imaging for sub-second whole brain FMRI and fast diffusion imaging. PLoS One 2010;5:e15710 CrossRef Medline 